\title{
COMMON FIXED POINT THEOREMS IN COMPATIBLE MAPPINGS OF TYPE (P*) OF GENERALIZED INTUITIONISTIC FUZZY METRIC SPACES
}

\author{
R.Muthuraj ${ }^{1} \&$ R.Pandiselvi ${ }^{2}$ \\ ${ }^{1}$ PG and Research Department of Mathematics, H.H.The Rajah's College, \\ Pudukkottai - 622 001, India. \\ ${ }^{2}$ Department of Mathematics, The Madura college, Madurai - 625 011, India.
}

\begin{abstract}
In this paper, we give some new definition of Compatible mappings of type $(P)$, type $(P-1)$ and type $(P-2)$ in intuitionistic generalized fuzzy metric spaces and prove Common fixed point theorems for six mappings under the conditions of compatible mappings of type $(P-1)$ and type $(P-2)$ in complete intuitionistic fuzzy metric spaces. Our results intuitionistically fuzzify the result of Muthuraj and Pandiselvi [15]
\end{abstract}

Mathematics subject classifications: $45 H 10,54 H 25$

\section{KEYWORDS}

Intuitionistic fuzzy metric spaces, compatible mappings of type $(P)$, type $(P-1)$ and type $(P-2)$, common fixed point.

\section{INTRODUCTION}

The Concept of fuzzy set was introduced by Zadeh [23] in 1965 .Following the concept of fuzzy sets, Deng [6] Kaleva and Seikalla [12] and kramosil and Michalek [13] introduced the concept of fuzzy metric space, George and Veeramani [7] modified the concept of fuzzy metric space introduced by kramosil and Michalek [13] .

Further, Sedghi and Shobe [19] defined $\mathcal{M}$-fuzzy metric space and proved a common fixed point theorem in it. Jong Seo Park [15] introduced the concept of semi compatible and Weak Compatible maps in fuzzy metric space and proved some fixed point theorems satisfying certain conditions in $\mathcal{M}$-fuzzy metric spaces.

As a generalization of fuzzy sets, Atanassov [1] introduced and studied the concept of intuitionistic fuzzy sets. Using the idea of intuitionistic fuzzy sets Park [16] defined the notion of intuitionistic fuzzy metric space with the help of continuous t- norm and continuous t- conorm as a generalization of fuzzy metric space, George and Veeramani [8] had showed that every metric induces an intuitionistic fuzzy metric and found a necessary and sufficient conditions for an intuitionistic fuzzy metric space to be complete. Choudhary [4] introduced mutually contractive sequence of self maps and proved a fixed point theorem. Kramaosil and Michalek [13] introduced the notion of Cauchy sequences in an intuitionistic fuzzy metric space and proved the well known fixed point theorem of Banach[2]. Turkoglu et al [22] gave the generalization of Jungck's[11] Common fixed point theorem to intuitionistic fuzzy metric spaces.

DOI : 10.5121/mathsj.2015.2301 
In this paper, we extend the result of common fixed point theorem for compatible mappings of type (P-1) and type (P-2) in intuitionistic fuzzy metric space and prove common fixed point theorem of type (P-1) and type (P-2) in intuitionistic fuzzy metric spaces, we also give an example to validate our main theorem. Our results intuitionistically fuzzify the result of Muthuraj and Pandiselvi [15].

\section{PRELIMINARIES}

We start with the following definitions.

\section{Definition 2.1}

A binary operation $*:[0,1] \times[0,1] \rightarrow[0,1]$ is said to be a continuous t-norm if $*$ is satisfies the following conditions.

(i) $*$ is commutative and associative,

(ii) $*$ is continuous,

(iii) $\quad \mathrm{a} * 1=\mathrm{a}$ for all $\mathrm{a} \in[0,1]$,

(iv) $\mathrm{a} * \mathrm{~b} \leq \mathrm{c} * \mathrm{~d}$ whenever $\mathrm{a} \leq \mathrm{c}$ and $\mathrm{b} \leq \mathrm{d}$ for all $\mathrm{a}, \mathrm{b}, \mathrm{c}, \mathrm{d} \in[0,1]$.

\section{Definition 2.2}

A binary operation $\diamond:[0,1] \times[0,1] \rightarrow[0,1]$ is said to be a continuous t-conorm if $\diamond$ satisfies the following conditions :

(i) $\nabla$ is commutative and associative,

(ii) $\diamond$ is continuous,

(iii) $\quad \mathrm{a} \diamond 0=\mathrm{a}$ for all $\mathrm{a} \in[0,1]$,

(iv) $\quad \mathrm{a} \diamond \mathrm{b} \leq \mathrm{c} \diamond \mathrm{d}$ whenever $\mathrm{a} \leq \mathrm{c}$ and $\mathrm{b} \leq \mathrm{d}$ for all $\mathrm{a}, \mathrm{b}, \mathrm{c}, \mathrm{d} \in[0,1]$.

\section{Definition 2.3}

A 5-tuple $(\mathrm{X}, \mathcal{M}, \mathcal{N}, *, \diamond)$ is called an intuitionistic fuzzy metric space if $\mathrm{X}$ is an arbitrary set, * is a continuous t-norm, $\diamond$ a continuous t-conorm and $\mathcal{M}, \mathcal{N}$ are fuzzy sets on $\mathrm{X}^{3} \times(0, \infty)$, satisfying the following conditions, for each $\mathrm{x}, \mathrm{y}, \mathrm{z}, \mathrm{a} \in \mathrm{X}$ and

$\mathrm{t}, \mathrm{s}>0$,
a) $\mathcal{M}(\mathrm{x}, \mathrm{y}, \mathrm{z}, \mathrm{t})+\mathcal{N}(\mathrm{x}, \mathrm{y}, \mathrm{z}, \mathrm{t}) \leq 1$.
b) $\mathcal{M}(\mathrm{x}, \mathrm{y}, \mathrm{z}, \mathrm{t})>0$.
c) $\mathcal{M}(\mathrm{x}, \mathrm{y}, \mathrm{z}, \mathrm{t})=1$ if and only if $\mathrm{x}=\mathrm{y}=\mathrm{z}$.
d) $\mathcal{M}(\mathrm{x}, \mathrm{y}, \mathrm{z}, \mathrm{t})=\mathcal{M}(\mathrm{p}\{\mathrm{x}, \mathrm{y}, \mathrm{z}\}, \mathrm{t})$ where $\mathrm{p}$ is a permutation function,
e) $\mathcal{M}(\mathrm{x}, \mathrm{y}, \mathrm{a}, \mathrm{t}) * \mathcal{M}(\mathrm{a}, \mathrm{z}, \mathrm{z}, \mathrm{s}) \leq \mathcal{M}(\mathrm{x}, \mathrm{y}, \mathrm{z}, \mathrm{t}+\mathrm{s})$
f) $\mathcal{M}(\mathrm{x}, \mathrm{y}, \mathrm{z}):(0, \infty) \rightarrow[0,1]$ is continuous
g) $\mathcal{N}(\mathrm{x}, \mathrm{y}, \mathrm{z}, \mathrm{t})>0$
h) $\mathcal{N}(\mathrm{x}, \mathrm{y}, \mathrm{z}, \mathrm{t})=0$, if and only if $\mathrm{x}=\mathrm{y}=\mathrm{z}$,
i) $\mathcal{N}(\mathrm{x}, \mathrm{y}, \mathrm{z}, \mathrm{t}=\mathcal{N}(\mathrm{p}\{\mathrm{x}, \mathrm{y}, \mathrm{z}\}, \mathrm{t})$ where $\mathrm{p}$ is a permutation function,
j) $\mathcal{N}(\mathrm{x}, \mathrm{y}, \mathrm{a}, \mathrm{t}) \diamond \mathcal{N}(\mathrm{a}, \mathrm{z}, \mathrm{z}, \mathrm{s}) \geq \mathcal{N}(\mathrm{x}, \mathrm{y}, \mathrm{z}, \mathrm{t}+\mathrm{s})$,
k) $\mathcal{N}(\mathrm{x}, \mathrm{y}, \mathrm{z}, \cdot):(0, \infty) \rightarrow[0,1]$ is continuous.

Then $(\mathcal{M}, \mathcal{N})$ is called an intuitionistic fuzzy metric on $\mathrm{X}$. 


\section{Example 2.4}

Let $\mathrm{X}=\mathrm{R}$, and $\mathcal{M}(\mathrm{x}, \mathrm{y}, \mathrm{z}, \mathrm{t})=\frac{\mathrm{t}}{\mathrm{t}+|\mathrm{x}-\mathrm{y}|+|\mathrm{y}-\mathrm{z}|+|\mathrm{z}-\mathrm{x}|}, \mathcal{N}(\mathrm{x}, \mathrm{y}, \mathrm{z}, \mathrm{t})=\frac{|\mathrm{x}-\mathrm{y}|+|\mathrm{y}-\mathrm{z}|+|\mathrm{z}-\mathrm{x}|}{\mathrm{t}+|\mathrm{x}-\mathrm{y}|+|\mathrm{y}-\mathrm{z}|+|\mathrm{z}-\mathrm{x}|}$ for every $\mathrm{x}$, $\mathrm{y}, \mathrm{z}$ and $\mathrm{t}>0$, let $\mathrm{A}$ and $\mathrm{B}$ defined as $\mathrm{Ax}=2 \mathrm{x}+1, \mathrm{Bx}=\mathrm{x}+2$, consider the sequence $\mathrm{x}_{\mathrm{n}}=\frac{1}{\mathrm{n}}+1, \mathrm{n}$ $=1,2, \ldots$ Thus we have

$\lim _{\mathrm{n} \rightarrow \infty} \mathcal{M}\left(\mathrm{Ax}_{\mathrm{n}}, 3,3, \mathrm{t}\right)=\lim _{\mathrm{n} \rightarrow \infty} \mathcal{M}\left(\mathrm{Bx}_{\mathrm{n}}, 3,3, \mathrm{t}\right)=1$ and

$\lim _{\mathrm{n} \rightarrow \infty} \mathcal{N}\left(\mathrm{Ax}_{\mathrm{n}}, 3,3, \mathrm{t}\right)=\lim _{\mathrm{n} \rightarrow \infty} \mathcal{N}\left(\mathrm{Bx}_{\mathrm{n}}, 3,3, \mathrm{t}\right)=0$, for every $\mathrm{t}>0$

Then A and B satisfying the property (E).

\section{Definition 2.5}

Let $(\mathrm{X}, \mathcal{M}, \mathcal{N}, *, \diamond)$ be an intuitionistic fuzzy metric space and $\left\{\mathrm{x}_{\mathrm{n}}\right\}$ be a sequence in $\mathrm{X}$.

a) $\left\{x_{n}\right\}$ is said to be converges to a point $\mathrm{x} \in \mathrm{X}$, if $\lim _{\mathrm{n} \rightarrow \infty} \mathcal{M}\left(\mathrm{x}, \mathrm{x}, \mathrm{x}_{\mathrm{n}}, \mathrm{t}\right)=1$ and $\lim _{\mathrm{n} \rightarrow \infty} \mathcal{N}\left(\mathrm{x}, \mathrm{x}, \mathrm{x}_{\mathrm{n}}, \mathrm{t}\right)=0$, for all $\mathrm{t}>0$.

b) $\left\{\mathrm{x}_{\mathrm{n}}\right\}$ is called Cauchy sequence if $\lim _{\mathrm{n} \rightarrow \infty} \mathcal{M}\left(\mathrm{x}_{\mathrm{n}+\mathrm{p}}, \mathrm{x}_{\mathrm{n}+\mathrm{p}}, \mathrm{x}_{\mathrm{n}}, \mathrm{t}\right)=1$ and $\lim _{n \rightarrow \infty} \mathcal{N}\left(x_{n+p}, x_{n+p}, x_{n}, t\right)=0$ for all $t>0$ and $p>0$.

c) An intuitionistic fuzzy metric space in which every Cauchy sequence is convergent is said to be complete.

\section{Lemma 2.6}

Let $(\mathrm{X}, \mathcal{M}, \mathcal{N}, *, \diamond)$ be an intuitionistic fuzzy metric space. Then $\mathcal{M}(\mathrm{x}, \mathrm{y}, \mathrm{z}, \mathrm{t})$ and $\mathcal{N}(\mathrm{x}, \mathrm{y}, \mathrm{z}, \mathrm{t})$ are non-decreasing with respect to $\mathrm{t}$, for all $\mathrm{x}, \mathrm{y}, \mathrm{z}$ in $\mathrm{X}$.

\section{Proof}

By definition 2.3, for each $\mathrm{x}, \mathrm{y}, \mathrm{z}, \mathrm{a} \in \mathrm{X}$ and $\mathrm{t}, \mathrm{s}>0$

we have $\mathcal{M}(\mathrm{x}, \mathrm{y}, \mathrm{a}, \mathrm{t}) * \mathcal{M}(\mathrm{a}, \mathrm{z}, \mathrm{z}, \mathrm{s}) \leq \mathcal{M}(\mathrm{x}, \mathrm{y}, \mathrm{z}, \mathrm{t}+\mathrm{s})$. If we set $\mathrm{a}=\mathrm{z}$,

we get $\mathcal{M}(\mathrm{z}, \mathrm{y}, \mathrm{z}, \mathrm{t}) * \mathcal{M}(\mathrm{z}, \mathrm{z}, \mathrm{z}, \mathrm{s}) \leq \mathcal{M}(\mathrm{x}, \mathrm{y}, \mathrm{z}, \mathrm{t}+\mathrm{s})$, that is

$\mathcal{M}(\mathrm{x}, \mathrm{y}, \mathrm{z}, \mathrm{t}+\mathrm{s}) \geq \mathcal{M}(\mathrm{x}, \mathrm{y}, \mathrm{z}, \mathrm{t})$.

Similarly, $\mathcal{N}(\mathrm{x}, \mathrm{y}, \mathrm{a}, \mathrm{t}) \diamond \mathcal{N}(\mathrm{a}, \mathrm{z}, \mathrm{z}, \mathrm{s}) \geq \mathcal{N}(\mathrm{x}, \mathrm{y}, \mathrm{z}, \mathrm{t}+\mathrm{s})$, for each $\mathrm{x}, \mathrm{y}, \mathrm{z}, \mathrm{a} \in \mathrm{X}$ and

$\mathrm{t}, \mathrm{s}>0$, by definition of $(\mathrm{X}, \mathcal{N}, \diamond)$. If we set $\mathrm{a}=\mathrm{z}$, we get

$\mathcal{N}(\mathrm{x}, \mathrm{y}, \mathrm{z}, \mathrm{t}) \diamond \mathcal{N}(\mathrm{z}, \mathrm{z}, \mathrm{z}, \mathrm{s}) \geq \mathcal{N}(\mathrm{x}, \mathrm{y}, \mathrm{z}, \mathrm{t}+\mathrm{s})$

that is $\mathcal{N}(\mathrm{x}, \mathrm{y}, \mathrm{z}, \mathrm{t}+\mathrm{s}) \leq \mathcal{N}(\mathrm{x}, \mathrm{y}, \mathrm{z}, \mathrm{t})$.Hence in IFMS $(\mathrm{X}, \mathcal{M}, \mathcal{N}, *, \diamond)$,

$\mathcal{M}(\mathrm{x}, \mathrm{y}, \mathrm{z}, \mathrm{t})$ and $\mathcal{N}(\mathrm{x}, \mathrm{y}, \mathrm{z}, \mathrm{t})$ are non-decreasing with respect to $\mathrm{t}$, for all $\mathrm{x}, \mathrm{y}, \mathrm{z}$ in $\mathrm{X}$. 


\section{COMPATIBLE MAPPINGS OF TYPE}

\section{Definition 3.1}

Let $\mathrm{A}$ and $\mathrm{S}$ be self mappings from an intuitionistic fuzzy metric space $(\mathrm{X}, \mathcal{M}, \mathcal{N}, *, \diamond)$ into itself. Then the mappings are said to be compatible if

$\lim _{\mathrm{n} \rightarrow \infty} \mathcal{M}\left(\mathrm{ASx}_{\mathrm{n}}, \mathrm{SAx}_{\mathrm{n}}, \mathrm{SAx}_{\mathrm{n}}, \mathrm{t}\right)=1$ and

$\lim _{n \rightarrow \infty} \mathcal{N}\left(\mathrm{ASx}_{\mathrm{n}}, \mathrm{SAx}_{\mathrm{n}}, \mathrm{SAx}_{\mathrm{n}}, \mathrm{t}\right)=0$, for all $\mathrm{t}>0$ whenever $\left\{\mathrm{x}_{\mathrm{n}}\right\}$ is a sequence in $\mathrm{X}$ such that $\lim _{\mathrm{n} \rightarrow \infty} \mathrm{Ax}_{\mathrm{n}}$ $=\lim _{\mathrm{n} \rightarrow \infty} S \mathrm{x}_{\mathrm{n}}=\mathrm{z}$ for some $\mathrm{z} \in \mathrm{X}$.

\section{Definition 3.2}

Let $\mathrm{A}$ and $\mathrm{S}$ be self mappings from an intuitionistic fuzzy metric space $(\mathrm{X}, \mathcal{M}, \mathcal{N}, *, \diamond)$ into itself. Then the mappings are said to be compatible of type (P), if

$\lim _{\mathrm{n} \rightarrow \infty} \mathcal{M}\left(\mathrm{AAx}_{\mathrm{n}}, \mathrm{SSx}_{\mathrm{n}}, \mathrm{SSx}_{\mathrm{n}}, \mathrm{t}\right)=1$ and $\lim _{\mathrm{n} \rightarrow \infty} \mathcal{N}\left(\mathrm{AAx}_{\mathrm{n}}, \mathrm{SSx}_{\mathrm{n}}, \mathrm{SSx}_{\mathrm{n}}, \mathrm{t}\right)=0$ for all $\mathrm{t}>0$, whenever $\left\{x_{n}\right\}$ is a sequence in $X$ such that $\lim _{n \rightarrow \infty} A x_{n}=\lim _{n \rightarrow \infty} S x_{n}=z$ for some $z \in X$.

\section{Definition 3.3}

Let $\mathrm{A}$ and $\mathrm{S}$ be self mappings from an intuitionistic fuzzy metric space $(\mathrm{X}, \mathcal{M}, \mathcal{N}, *, \diamond)$ into itself. Then the mappings are said to be R-Weakly commuting of type $(\mathrm{P})$, if there exists some $\mathrm{R}>0$, such that $\mathcal{M}$ (AAx, SSx, SSx, t) $\geq \mathcal{M}\left(\mathrm{Ax}, \mathrm{Sx}, \mathrm{Sx}, \frac{\mathrm{t}}{\mathrm{R}}\right)$,

$\mathcal{N}(\mathrm{AAx}, \mathrm{SSx}, \mathrm{SSx}, \mathrm{t}) \leq \mathcal{N}\left(\mathrm{Ax}, \mathrm{Sx}, \mathrm{Sx}, \frac{\mathrm{t}}{\mathrm{R}}\right)$, for all $\mathrm{x}$ in $\mathrm{X}$ and $\mathrm{t}>0$

\section{Definition 3.4}

Let $\mathrm{A}$ and $\mathrm{S}$ be self mappings from an intuitionistic fuzzy metric space $(\mathrm{X}, \mathcal{M}, \mathcal{N}, *, \diamond)$ into itself. Then the mappings are said to be compatible of type (P-1) if

$\lim _{\mathrm{n} \rightarrow \infty} \mathcal{M}\left(\mathrm{SAx}_{\mathrm{n}}, \mathrm{AAx}_{\mathrm{n}}, \mathrm{AAx}_{\mathrm{n}}, \mathrm{t}\right)=1$ and $\lim _{\mathrm{n} \rightarrow \infty} \mathcal{N}\left(\mathrm{SAx}_{\mathrm{n}}, \mathrm{AAx}_{\mathrm{n}}, \mathrm{AAx}_{\mathrm{n}}, \mathrm{t}\right)=0$ for all $\mathrm{t}>0$, whenever $\left\{x_{n}\right\}$ is a sequence in $X$ such that $\lim _{n \rightarrow \infty} A x_{n}=\lim _{n \rightarrow \infty} S x_{n}=z$ for some $z \in X$.

\section{Definition 3.5}

Let $\mathrm{A}$ and $\mathrm{S}$ be self mappings from an intuitionistic fuzzy metric space $(\mathrm{X}, \mathcal{M}, \mathcal{N}, *, \diamond)$ into itself. Then the mappings are said to be compatible of type (P-2) if

$\lim _{\mathrm{n} \rightarrow \infty} \mathcal{M}\left(\mathrm{AAx}_{\mathrm{n}}, \mathrm{SSx}_{\mathrm{n}}, \mathrm{SSx}_{\mathrm{n}}, \mathrm{t}\right)=1$ and $\lim _{\mathrm{n} \rightarrow \infty} \mathcal{N}\left(\mathrm{AAx}_{\mathrm{n}}, \mathrm{SSx}_{\mathrm{n}}, \mathrm{SSx}_{\mathrm{n}}, \mathrm{t}\right)=0$ for all $\mathrm{t}>0$ whenever $\left\{\mathrm{x}_{\mathrm{n}}\right\}$ is a sequence in $X$ such that $\lim _{n \rightarrow \infty} A x_{n}=\lim _{n \rightarrow \infty} S x_{n}=z$ for some $z \in X$. 


\section{Proposition 3.6}

Let $\mathrm{A}$ and $\mathrm{S}$ be self mappings from an intuitionistic fuzzy metric space $(\mathrm{X}, \mathcal{M}, \mathcal{N}, *, \diamond)$ into itself.

a) If $\mathrm{A}$ is continuous map then the pair of mappings (A, S) is compatible of type (P-1) if and only if $\mathrm{A}$ and $\mathrm{S}$ are compatible.

b) If $S$ is a continuous map then the pair of mappings $(\mathrm{A}, \mathrm{S})$ is compatible of type (P-2) if and only if A and S are compatible.

\section{Proof}

a) Let $\lim _{n \rightarrow \infty} A x_{n}=\lim _{n \rightarrow \infty} S x_{n}=z$ for some $z \in X$, and let the pair (A,S) be compatible of type (P-1). Since A is continuous, we have $\lim _{n \rightarrow \infty} \mathrm{ASx}_{n}=\mathrm{Az}$ and $\lim _{\mathrm{n} \rightarrow \infty} \mathrm{AAx}_{\mathrm{n}}=\mathrm{Az}$. Therefore it follows that

$$
\begin{aligned}
\mathcal{M}\left(\mathrm{SAx}_{\mathrm{n}}, \mathrm{ASx}_{\mathrm{n}}, \mathrm{ASx}_{\mathrm{n}}, \mathrm{t}\right) & \geq \mathcal{M}\left(\mathrm{SAx}_{\mathrm{n}}, \mathrm{AAx}_{\mathrm{n}}, \mathrm{AAx}_{\mathrm{n}}, \frac{\mathrm{t}}{2}\right) \\
& * \mathcal{M}\left(\mathrm{AAx}_{\mathrm{n}}, \mathrm{ASx}_{\mathrm{n}}, \mathrm{ASx}_{\mathrm{n}}, \frac{\mathrm{t}}{2}\right) \text { and } \\
\mathcal{N}\left(\mathrm{SAx}_{\mathrm{n}}, \mathrm{ASx}_{\mathrm{n}}, \mathrm{ASx}_{\mathrm{n}}, \mathrm{t}\right) \leq & \mathcal{N}\left(\mathrm{SAx}_{\mathrm{n}}, \mathrm{AAx}_{\mathrm{n}}, \mathrm{AAx}_{\mathrm{n}}, \frac{\mathrm{t}}{2}\right) \\
& \diamond \mathcal{N}\left(\mathrm{AAx}_{\mathrm{n}}, \mathrm{ASx}_{\mathrm{n}}, \mathrm{ASx}_{\mathrm{n}}, \frac{\mathrm{t}}{2}\right)
\end{aligned}
$$

yields $\lim _{\mathrm{n} \rightarrow \infty} \mathcal{M}\left(\mathrm{SAx}_{\mathrm{n}}, \mathrm{ASx}_{\mathrm{n}}, \mathrm{ASx}_{\mathrm{n}}, \mathrm{t}\right) \geq 1 * 1=1$ and $\lim _{\mathrm{n} \rightarrow \infty} \mathcal{N}\left(\mathrm{SAx}_{\mathrm{n}}, \mathrm{ASx}_{\mathrm{n}}, \mathrm{ASx}_{\mathrm{n}}, \mathrm{t}\right) \leq 0 \diamond 0=0$ and so the mappings A and $\mathrm{S}$ are compatible. Now, let $\mathrm{A}$ and $\mathrm{S}$ be compatible. Therefore it follows that

$$
\begin{aligned}
& \mathcal{M}\left(\mathrm{SAx}_{\mathrm{n}}, \mathrm{AAx}_{\mathrm{n}}, \mathrm{AAx}_{\mathrm{n}}, \mathrm{t}\right) \geq \mathcal{M}\left(\mathrm{SAx}_{\mathrm{n}}, \mathrm{ASx}_{\mathrm{n}}, \mathrm{ASx}_{\mathrm{n}}, \frac{\mathrm{t}}{2}\right) \\
& * \mathcal{M}\left(\mathrm{ASx}_{\mathrm{n}}, \quad \mathrm{AAx}_{\mathrm{n}}, \quad \mathrm{AAx}_{\mathrm{n}}, \quad \frac{\mathrm{t}}{2}\right) \\
& \mathcal{N}\left(\mathrm{SAx}_{\mathrm{n}}, \mathrm{AAx}_{\mathrm{n}}, \mathrm{AAx}_{\mathrm{n}}, \mathrm{t}\right) \leq \mathcal{N}\left(\mathrm{SAx}_{\mathrm{n}}, \mathrm{ASx}_{\mathrm{n}}, \mathrm{ASx}_{\mathrm{n}}, \frac{\mathrm{t}}{2}\right) \\
& \diamond \mathcal{N}\left(\mathrm{ASx}_{\mathrm{n}}, \quad \mathrm{AAx}_{\mathrm{n}}, \quad \mathrm{AAx}_{\mathrm{n}}, \quad \frac{\mathrm{t}}{2}\right) \\
& \lim _{\mathrm{n} \rightarrow \infty} \mathcal{N}\left(\mathrm{SAx}_{\mathrm{n}}, \mathrm{AAx}_{\mathrm{n}}, \mathrm{AAx}_{\mathrm{n}}, \mathrm{t}\right) \leq 0 \diamond 0=0 \text { and } \lim _{\mathrm{N} \rightarrow \infty} \mathcal{M}\left(\mathrm{SAx}_{\mathrm{n}}, \mathrm{AAx}_{\mathrm{n}}, \mathrm{AAx}_{\mathrm{n}}, \mathrm{t}\right) \geq 1 * 1=1 \text { and }
\end{aligned}
$$

so that pair of mappings $(\mathrm{A}, \mathrm{S})$ are compatible of type (P-1). 
Applied Mathematics and Sciences: An International Journal (MathSJ ), Vol. 2, No. 3, September 2015

b) Let $\lim _{\mathrm{n} \rightarrow \infty} \mathrm{Sx}_{\mathrm{n}}=\lim _{\mathrm{n} \rightarrow \infty} A \mathrm{x}_{\mathrm{n}}=\mathrm{z}$ for some $\mathrm{z}$ in $\mathrm{X}$ and let the pair $(\mathrm{A}, \mathrm{S})$ be compatible of type (P-2). Since $S$ is continuous, we have $\lim _{n \rightarrow \infty} S A x_{n}=S z$ and

$\lim _{n \rightarrow \infty} \mathrm{SSx}_{\mathrm{n}}=\mathrm{Sz}$. Therefore it follows that

$$
\begin{gathered}
\mathcal{M}\left(\mathrm{SAx}_{\mathrm{n}}, \mathrm{ASx}_{\mathrm{n}}, \mathrm{ASx}_{\mathrm{n}}, \mathrm{t}\right) \geq \mathcal{M}\left(\mathrm{SAx}_{\mathrm{n}}, \mathrm{SSx}_{\mathrm{n}}, \mathrm{SSx}_{\mathrm{n}}, \frac{\mathrm{t}}{2}\right) \\
* \mathcal{M}\left(\mathrm{SSx}_{\mathrm{n}}, \mathrm{ASx}_{\mathrm{n}}, \mathrm{ASx}_{\mathrm{n}}, \frac{\mathrm{t}}{2}\right) \text { and } \\
\mathcal{N}\left(\mathrm{SAx}_{\mathrm{n}}, \mathrm{ASx}_{\mathrm{n}}, \mathrm{ASx}_{\mathrm{n}}, \mathrm{t}\right) \leq \mathcal{N}\left(\mathrm{SAx}_{\mathrm{n}}, \mathrm{SSx}_{\mathrm{n}}, \mathrm{SSx}_{\mathrm{n}}, \frac{\mathrm{t}}{2}\right) \\
\diamond \mathcal{N}\left(\mathrm{SSx}_{\mathrm{n}}, \mathrm{ASx}_{\mathrm{n}}, \mathrm{ASx}_{\mathrm{n}}, \frac{\mathrm{t}}{2}\right)
\end{gathered}
$$

yields $\lim _{\mathrm{n} \rightarrow \infty} \mathcal{M}\left(\mathrm{SAx}_{\mathrm{n}}, \mathrm{ASx}_{\mathrm{n}}, \mathrm{ASx}_{\mathrm{n}}, \mathrm{t}\right) \geq 1 * 1=1$ and

$\lim _{\mathrm{n} \rightarrow \infty} \mathcal{N}\left(\mathrm{SAx}_{\mathrm{n}}, \mathrm{ASx}_{\mathrm{n}}, \mathrm{ASx}_{\mathrm{n}}, \mathrm{t}\right) \leq 0 \diamond 0=0$ and so the mappings $\mathrm{A}$ and $\mathrm{S}$ are compatible.

Now let $\mathrm{A}$ and $\mathrm{S}$ be compatible. Then we have

$$
\begin{gathered}
\mathcal{M}\left(\mathrm{ASx}_{\mathrm{n}}, \mathrm{SSx}_{\mathrm{n}}, \mathrm{SSx}_{\mathrm{n}}, \mathrm{t}\right) \geq \mathcal{M}\left(\mathrm{ASx}_{\mathrm{n}}, \mathrm{SAx}_{\mathrm{n}}, \mathrm{SAx}_{\mathrm{n}}, \frac{\mathrm{t}}{2}\right) \\
* \mathcal{M}\left(\mathrm{SAx}_{\mathrm{n}}, \mathrm{SSx}_{\mathrm{n}}, \mathrm{SSx}_{\mathrm{n}}, \frac{\mathrm{t}}{2}\right) \text { and } \\
\mathcal{N}\left(\mathrm{ASx}_{\mathrm{n}}, \mathrm{SSx}_{\mathrm{n}}, \mathrm{SSx}_{\mathrm{n}}, \mathrm{t}\right) \leq \mathcal{N}\left(\mathrm{ASx}_{\mathrm{n}}, \mathrm{SAx}_{\mathrm{n}}, \mathrm{SAx}_{\mathrm{n}}, \frac{\mathrm{t}}{2}\right) \\
\diamond \mathcal{N}\left(\mathrm{SAx}_{\mathrm{n}}, \mathrm{SSx}_{\mathrm{n}}, \mathrm{SSx}_{\mathrm{n}}, \frac{\mathrm{t}}{2}\right)
\end{gathered}
$$

yields $\lim _{\mathrm{n} \rightarrow \infty} \mathcal{M}\left(\mathrm{ASx}_{\mathrm{n}}, \mathrm{SSx}_{\mathrm{n}}, \mathrm{SSx}_{\mathrm{n}}, \mathrm{t}\right) \geq 1 * 1=1$ and

$\lim _{\mathrm{n} \rightarrow \infty} \mathcal{N}\left(\mathrm{ASx}_{\mathrm{n}}, \mathrm{SSx}_{\mathrm{n}}, \mathrm{SSx}_{\mathrm{n}}, \mathrm{t}\right) \leq 0 \diamond 0=0$ and so the pair of mappings (A, S) are

compatible of type (P-2).

\section{Proposition 3.7}

Let $\mathrm{A}$ and $\mathrm{S}$ be self mappings from an intuitionistic fuzzy metric space $(\mathrm{X}, \mathcal{M}, \mathcal{N}, *, \diamond)$ into itself. If the pair $(\mathrm{A}, \mathrm{S})$ is compatible of type (P-2) and $\mathrm{Sz}=\mathrm{Az}$ for some $\mathrm{z} \in \mathrm{X}$. Then $\mathrm{ASz}=\mathrm{SSz}$.

\section{Proof:}

Let $\left\{x_{n}\right\}$ be a sequence in $X$ defined by $x_{n}=z$ for $n=1,2, \ldots$ and let $A z=S z$.

Then we have $\lim _{n \rightarrow \infty} S x_{n}=S z$ and $\lim _{n \rightarrow \infty} A x_{n}=A z$. Since the pair $(A, S)$ is compatible of type (P-2), we have

$$
\begin{gathered}
\mathcal{M}(\mathrm{ASz}, \mathrm{SSz}, \mathrm{SSz}, \mathrm{t})=\lim _{\mathrm{n} \rightarrow \infty} \mathcal{M}\left(\mathrm{ASx}_{\mathrm{n}}, \mathrm{SSx}_{\mathrm{n}}, \mathrm{SSx}_{\mathrm{n}}, \mathrm{t}\right)=1 \text { and } \\
\mathcal{N}(\mathrm{ASz}, \mathrm{SSz}, \mathrm{SSz}, \mathrm{t})=\lim _{\mathrm{n} \rightarrow \infty} \mathcal{N}\left(\mathrm{ASx}_{\mathrm{n}}, \mathrm{SSx}_{\mathrm{n}}, \mathrm{SSx}_{\mathrm{n}}, \mathrm{t}\right)=0 .
\end{gathered}
$$

Hence ASz $=$ SSz . 


\section{Proposition 3.8}

Let $\mathrm{A}$ and $\mathrm{S}$ self mappings from an intuitionistic fuzzy metric space $(\mathrm{X}, \mathcal{M}, \mathcal{N}, *, \diamond)$ with $\mathrm{t} * \mathrm{t} \geq$ $\mathrm{t}$ and $(1-\mathrm{t}) \diamond(1-\mathrm{t}) \leq 1-\mathrm{t}$ for all $\mathrm{t} \in[0,1]$ if the pair $(\mathrm{A}, \mathrm{S})$ are compatible of type $(\mathrm{p}-1)$ and $\mathrm{Ax}_{\mathrm{n}}$, $\mathrm{Sx}_{\mathrm{n}} \rightarrow \mathrm{z}$ for some $\mathrm{z}$ in $\mathrm{X}$ and a sequence $\left\{\mathrm{x}_{\mathrm{n}}\right\}$ in $\mathrm{X}$.

Then $\mathrm{AAx}_{\mathrm{n}} \rightarrow \mathrm{Sz}$, if $\mathrm{S}$ is continuous at $\mathrm{z}$.

\section{Proof}

Since $S$ is continuous at $z$, we have $\mathrm{SAx}_{\mathrm{n}} \rightarrow \mathrm{Sz}$. Since the pair (A, S) are compatible of type (P-1), we have $\mathcal{M}\left(\mathrm{SAx}_{\mathrm{n}}, \mathrm{AAx}_{\mathrm{n}}, \mathrm{AAx}_{\mathrm{n}}, \mathrm{t}\right) \rightarrow 1$ as $\mathrm{n} \rightarrow \infty$. It follows that

$$
\begin{aligned}
& \mathcal{M}\left(\mathrm{Sz}, \mathrm{AAx}_{\mathrm{n}}, \mathrm{AAx}_{\mathrm{n}}, \mathrm{t}\right) \geq \mathcal{M}\left(\mathrm{Sz}, \mathrm{SAx}_{\mathrm{n}}, \mathrm{SAx}_{\mathrm{n}}, \frac{\mathrm{t}}{2}\right) * \mathcal{M}\left(\mathrm{SAx}_{\mathrm{n}}, \mathrm{AAx}_{\mathrm{n}}, \mathrm{AAx}_{\mathrm{n}}, \frac{\mathrm{t}}{2}\right) \text { and } \\
& \mathcal{N}\left(\mathrm{Sz}_{\mathrm{AAx}}, \mathrm{AAx}_{\mathrm{n}}, \mathrm{t}\right) \leq \mathcal{N}\left(\mathrm{Sz}, \mathrm{SAx}_{\mathrm{n}}, \mathrm{SAx}_{\mathrm{n}}, \frac{\mathrm{t}}{2}\right) * \mathcal{N}\left(\mathrm{SAx}_{\mathrm{n}}, \mathrm{AAx}_{\mathrm{n}}, \mathrm{AAx}_{\mathrm{n}}, \frac{\mathrm{t}}{2}\right) \text { yield } \\
& \mathcal{M}\left(\mathrm{Sz}, \mathrm{AAx}_{\mathrm{n}}, \mathrm{AAx}_{\mathrm{n}}, \mathrm{t}\right) \geq 1 * 1=1 \text { and } \\
& \mathcal{N}\left(\mathrm{Sz}_{\mathrm{AAx}}, \mathrm{AAx}_{\mathrm{n}}, \mathrm{t}\right) \leq 0 \diamond 0=0 \text { and so }
\end{aligned}
$$

we have $\mathrm{AAx}_{\mathrm{n}} \rightarrow \mathrm{Sz}$ as $\mathrm{n} \rightarrow \infty$.

\section{Proposition 3.9}

Let $\mathrm{A}$ and $\mathrm{S}$ be self mappings from an intuitionistic fuzzy metric space $(\mathrm{X}, \mathcal{M}, \mathcal{N}, *, \diamond)$ with $\mathrm{t} * \mathrm{t} \geq$ $t$ and (1- t) $\diamond(1-t) \leq 1-t$ for $t \in[0,1]$. If the pair (A, S) are compatible of type (P - 2) and $A x_{n}$, $\mathrm{Sx}_{\mathrm{n}} \rightarrow \mathrm{z}$ for some $\mathrm{z}$ in $\mathrm{X}$ and sequence $\left\{\mathrm{x}_{\mathrm{n}}\right\}$ in $\mathrm{X}$. Then $\mathrm{SSx}_{\mathrm{n}} \rightarrow \mathrm{Az}$ if $\mathrm{A}$ is continuous at $\mathrm{z}$.

\section{Proof}

Since A is continuous at $\mathrm{z}$, we have $\mathrm{ASx}_{\mathrm{n}} \rightarrow \mathrm{Az}$. Since the pair $(\mathrm{A}, \mathrm{S})$ are compatible of type (P 2), we have $\mathcal{M}\left(\mathrm{ASx}_{\mathrm{n}}, \mathrm{SSx}_{\mathrm{n}}, \mathrm{SSx}_{\mathrm{n}}, \mathrm{t}\right) \rightarrow 1$ as $\mathrm{n} \rightarrow \infty$, it follows that

$$
\begin{gathered}
\mathcal{M}\left(\mathrm{Az}, \mathrm{SSx}_{\mathrm{n}}, \mathrm{SSx}_{\mathrm{n}}, \mathrm{t}\right) \geq \mathcal{M}\left(\mathrm{Az}, \mathrm{ASx}_{\mathrm{n}}, \mathrm{ASx}_{\mathrm{n}}, \frac{\mathrm{t}}{2}\right) * \mathcal{M}\left(\mathrm{ASx}_{\mathrm{n}}, \mathrm{SSx}_{\mathrm{n}}, \mathrm{SSx}_{\mathrm{n}}, \frac{\mathrm{t}}{2}\right) \text { and } \\
\mathcal{N}\left(\mathrm{Az}, \mathrm{SSx}_{\mathrm{n}}, \mathrm{SSx}_{\mathrm{n}}, \mathrm{t}\right) \leq \mathcal{N}\left(\mathrm{Az}, \mathrm{ASx}_{\mathrm{n}}, \mathrm{ASx}_{\mathrm{n}}, \frac{\mathrm{t}}{2}\right) \diamond \mathcal{N}\left(\mathrm{ASx}_{\mathrm{n}}, \mathrm{SSx}_{\mathrm{n}}, \mathrm{SSx}_{\mathrm{n}}, \frac{\mathrm{t}}{2}\right) \text { yield } \\
\lim _{\mathrm{n} \rightarrow \infty} \mathcal{M}\left(\mathrm{Az}, \mathrm{SSx}_{\mathrm{n}}, \mathrm{SSx}_{\mathrm{n}}, \mathrm{t}\right) \geq 1 * 1=1 \text { and } \\
\lim _{\mathrm{n} \rightarrow \infty} \mathcal{N}\left(\mathrm{Az}, \mathrm{SSx}_{\mathrm{n}}, \mathrm{SSx}_{\mathrm{n}}, \mathrm{t}\right) \leq 0 \diamond 0=0 \text { and so }
\end{gathered}
$$

we have $\mathrm{SSx}_{\mathrm{n}} \rightarrow \mathrm{Az}$ as $\mathrm{n} \rightarrow \infty$. 


\section{MAIN RESULTS}

\section{Theorem 4.1}

Let $(\mathrm{X}, \mathcal{M}, \mathcal{N}, *, \diamond)$ be a complete generalized intuitionistic fuzzy metric space and let $\mathrm{A}, \mathrm{B}, \mathrm{P}, \mathrm{Q}$, $\mathrm{S}$ and $\mathrm{T}$ be self mappings of $\mathrm{X}$ satisfying the following conditions.

(i) $\quad \mathrm{P}(\mathrm{X}) \subseteq \mathrm{ST}(\mathrm{X}), \mathrm{Q}(\mathrm{X}) \subseteq \mathrm{AB}(\mathrm{X})$

(ii) The pair $(\mathrm{P}, \mathrm{AB})$ and $(\mathrm{Q}, \mathrm{ST})$ are compatible mappings of type $(\mathrm{P})$

(iii) $\quad \mathrm{ST}$ is continuous

(iv) $\mathcal{M}(\mathrm{Px}, \mathrm{Qz}, \mathrm{Qz}, \mathrm{qt}) \geq \min \{\mathcal{M}(\mathrm{ABx}, \mathrm{Py}, \mathrm{Qy}, \mathrm{t}), \mathcal{M}(\mathrm{ABx}, \mathrm{Py}, \mathrm{STz}, \mathrm{t})$,

$\mathcal{M}(\mathrm{Qy}, \mathrm{STz}, \mathrm{Py}, \mathrm{t}), \mathcal{M}(\mathrm{ABx}, \mathrm{Qy}, \mathrm{STz}, \mathrm{t})\}$ and

$\mathcal{N}(\mathrm{Px}, \mathrm{Qz}, \mathrm{Qz}, \mathrm{qt}) \leq \max \{\mathcal{N}(\mathrm{ABx}, \mathrm{Py}, \mathrm{Qy}, \mathrm{t}), \mathcal{N}(\mathrm{ABx}, \mathrm{Py}, \mathrm{STz}, \mathrm{t})$,

$\mathcal{N}(\mathrm{Qy}, \mathrm{STz}, \mathrm{Py}, \mathrm{t}), \mathcal{N}(\mathrm{ABx}, \mathrm{Qy}, \mathrm{STz}, \mathrm{t})\}$

then the mappings $\mathrm{P}, \mathrm{Q}, \mathrm{AB}$ and $\mathrm{ST}$ have a unique common fixed point in $\mathrm{X}$.

\section{Proof}

Let $\mathrm{x}_{0}$ be any arbitrary point in $\mathrm{X}$. Thus we construct a sequence $\left\{\mathrm{y}_{\mathrm{n}}\right\}$ in $\mathrm{X}$ such that $\mathrm{y}_{2 \mathrm{n}-1}=\mathrm{STX}_{2 \mathrm{n}-1}=\mathrm{Px}_{2 \mathrm{n}-2}$ and $\mathrm{y}_{2 \mathrm{n}}=\mathrm{ABx}_{2 \mathrm{n}}=\mathrm{Qx}_{2 \mathrm{n}-1}$. Put $\mathrm{x}=\mathrm{x}_{2 \mathrm{n}-1}, \mathrm{y}=\mathrm{x}_{2 \mathrm{n}-1}, \mathrm{z}=\mathrm{x}_{2 \mathrm{n}}$.

$$
\begin{gathered}
\mathcal{M}\left(\mathrm{Px}_{2 \mathrm{n}-1}, \mathrm{Qx}_{2 \mathrm{n}}, \mathrm{Qx}_{2 \mathrm{n}}, \mathrm{qt}\right) \geq \min \left\{\begin{array}{c}
\mathcal{M}\left(\mathrm{ABx}_{2 \mathrm{n}-1}, \mathrm{Px}_{2 \mathrm{n}-1}, \mathrm{Qx}_{2 \mathrm{n}-1}, \mathrm{t}\right) \\
\mathcal{M}\left(\mathrm{ABx}_{2 \mathrm{n}-1}, \mathrm{Px}_{2 \mathrm{n}-1}, \mathrm{STx}_{2 \mathrm{n}}, \mathrm{t}\right), \\
\mathcal{M}\left(\mathrm{Qx}_{2 \mathrm{n}-1}, \mathrm{STx}_{2 \mathrm{n}}, \mathrm{Px}_{2 \mathrm{n}-1}, \mathrm{t}\right), \\
\mathcal{M}\left(\mathrm{ABx}_{2 \mathrm{n}-1}, \mathrm{Qx}_{2 \mathrm{n}-1}, \mathrm{STx}_{2 \mathrm{n}}, \mathrm{t}\right)
\end{array}\right\} \\
\mathcal{M}\left(\mathrm{y}_{2 \mathrm{n}}, \mathrm{y}_{2 \mathrm{n}+1}, \mathrm{y}_{2 \mathrm{n}+1}, \mathrm{qt}\right) \geq \min \left\{\begin{array}{c}
\mathcal{M}\left(\mathrm{y}_{2 \mathrm{n}-1}, \mathrm{y}_{2 \mathrm{n}}, \mathrm{y}_{2 \mathrm{n}}, \mathrm{t}\right), \\
\mathcal{M}\left(\mathrm{y}_{2 \mathrm{n}-1}, \mathrm{y}_{2 \mathrm{n}}, \mathrm{y}_{2 \mathrm{n}}, \mathrm{t}\right), \\
\mathcal{M}\left(\mathrm{y}_{2 \mathrm{n}}, \mathrm{y}_{2 \mathrm{n}}, \mathrm{y}_{2 \mathrm{n}}, \mathrm{t}\right), \\
\mathcal{M}\left(\mathrm{y}_{2 \mathrm{n}-1}, \mathrm{y}_{2 \mathrm{n}}, \mathrm{y}_{2 \mathrm{n}}, \mathrm{t}\right)
\end{array}\right\} \\
\mathcal{M}\left(\mathrm{y}_{2 \mathrm{n}}, \mathrm{y}_{2 \mathrm{n}+1}, \mathrm{y}_{2 \mathrm{n}+1}, \mathrm{qt}\right) \geq \mathcal{M}\left(\mathrm{y}_{2 \mathrm{n}-1}, \mathrm{y}_{2 \mathrm{n}}, \mathrm{y}_{2 \mathrm{n}}, \mathrm{t}\right)
\end{gathered}
$$

This implies that $\mathcal{M}\left(\mathrm{y}_{2 n}, \mathrm{y}_{2 \mathrm{n}+1}, \mathrm{y}_{2 \mathrm{n}+1}, \mathrm{t}\right)$ is an increasing sequence of positive real numbers.

$$
\begin{aligned}
& \mathcal{N}\left(\mathrm{Px}_{2 \mathrm{n}-1}, \mathrm{Qx}_{2 \mathrm{n}}, \mathrm{Qx}_{2 \mathrm{n}}, \mathrm{qt}\right) \leq \max \left\{\begin{array}{c}
\mathcal{N}\left(\mathrm{ABx}_{2 \mathrm{n}-1}, \mathrm{Px}_{2 \mathrm{n}-1}, \mathrm{Qx}_{2 \mathrm{n}-1}, \mathrm{t}\right) \\
\mathcal{N}\left(\mathrm{ABx}_{2 \mathrm{n}-1}, \mathrm{Px}_{2 \mathrm{n}-1}, \mathrm{STx}_{2 \mathrm{n}}, \mathrm{t}\right) \\
\mathcal{N}\left(\mathrm{Qx}_{2 \mathrm{n}-1}, \mathrm{STx}_{2 \mathrm{n}}, \mathrm{Px}_{2 \mathrm{n}-1}, \mathrm{t}\right) \\
\mathcal{N}\left(\mathrm{ABx}_{2 \mathrm{n}-1}, \mathrm{Qx}_{2 \mathrm{n}-1}, \mathrm{STx}_{2 \mathrm{n}}, \mathrm{t}\right)
\end{array}\right\} \\
& \mathcal{N}\left(\mathrm{y}_{2 n}, \mathrm{y}_{2 n+1}, \mathrm{y}_{2 n+1}, \mathrm{qt}\right) \leq \max \left\{\begin{array}{c}
\mathcal{N}\left(\mathrm{y}_{2 n-1}, \mathrm{y}_{2 n}, \mathrm{y}_{2 n}, \mathrm{t}\right) \\
\mathcal{N}\left(\mathrm{y}_{2 n-1}, \mathrm{y}_{2 n}, \mathrm{y}_{2 n}, \mathrm{t}\right) \\
\mathcal{N}\left(\mathrm{y}_{2 n}, \mathrm{y}_{2 n}, \mathrm{y}_{2 n}, \mathrm{t}\right) \\
\mathcal{N}\left(\mathrm{y}_{2 n-1}, \mathrm{y}_{2 n}, \mathrm{y}_{2 n}, \mathrm{t}\right)
\end{array}\right\} \\
& \mathcal{N}\left(\mathrm{y}_{2 \mathrm{n}}, \mathrm{y}_{2 \mathrm{n}+1}, \mathrm{y}_{2 \mathrm{n}+1}, \mathrm{qt}\right) \leq \mathcal{N}\left(\mathrm{y}_{2 \mathrm{n}-1}, \mathrm{y}_{2 \mathrm{n}}, \mathrm{y}_{2 \mathrm{n}}, \mathrm{t}\right)
\end{aligned}
$$

This implies that $\mathcal{N}\left(\mathrm{y}_{2 n}, \mathrm{y}_{2 \mathrm{n}+1}, \mathrm{y}_{2 \mathrm{n}+1}, \mathrm{t}\right)$ is an decreasing sequence of positive real numbers. Now to prove that $\mathcal{M}\left(\mathrm{y}_{\mathrm{n}}, \mathrm{y}_{\mathrm{n}+1}, \mathrm{y}_{\mathrm{n}+1}, \mathrm{t}\right)$ converges to 1 as $\mathrm{n} \rightarrow \infty$ and $\mathcal{N}\left(\mathrm{y}_{2 \mathrm{n}}, \mathrm{y}_{2 \mathrm{n}+1}, \mathrm{y}_{2 \mathrm{n}+1}, \mathrm{t}\right)$ converges to 0 as $\mathrm{n} \rightarrow \infty$. By lemma 2.6, 


$$
\begin{gathered}
\mathcal{M}\left(\mathrm{y}_{\mathrm{n}}, \mathrm{y}_{\mathrm{n}+1}, \mathrm{y}_{\mathrm{n}+1}, \mathrm{t}\right) \geq \mathcal{M}\left(\mathrm{y}_{\mathrm{n}-1}, \mathrm{y}_{\mathrm{n}}, \mathrm{y}_{\mathrm{n}}, \frac{\mathrm{t}}{\mathrm{q}}\right) \geq \mathcal{M}\left(\mathrm{y}_{\mathrm{n}-2}, \mathrm{y}_{\mathrm{n}-1}, \mathrm{y}_{\mathrm{n}-1}, \frac{\mathrm{t}}{\mathrm{q}^{2}}\right) \\
\ldots \geq \mathcal{M}\left(\mathrm{y}_{0}, \mathrm{y}_{1}, \mathrm{y}_{1}, \frac{\mathrm{t}}{\mathrm{q}^{\mathrm{n}}}\right) \\
\text { Thus } \mathcal{M}\left(\mathrm{y}_{\mathrm{n}}, \mathrm{y}_{\mathrm{n}+1}, \mathrm{y}_{\mathrm{n}+1}, \mathrm{t}\right) \geq \mathcal{M}\left(\mathrm{y}_{0}, \mathrm{y}_{1}, \mathrm{y}_{1}, \frac{\mathrm{t}}{\mathrm{q}^{\mathrm{n}}}\right) \text { and } \\
\mathcal{N}\left(\mathrm{y}_{\mathrm{n}}, \mathrm{y}_{\mathrm{n}+1}, \mathrm{y}_{\mathrm{n}+1}, \mathrm{t}\right) \leq \mathcal{N}\left(\mathrm{y}_{\mathrm{n}-1}, \mathrm{y}_{\mathrm{n}}, \mathrm{y}_{\mathrm{n}}, \frac{\mathrm{t}}{\mathrm{q}}\right) \leq \mathcal{N}\left(\mathrm{y}_{\mathrm{n}-2}, \mathrm{y}_{\mathrm{n}-1}, \mathrm{y}_{\mathrm{n}-1}, \frac{\mathrm{t}}{\mathrm{q}^{2}}\right) \\
\ldots \leq \mathcal{N}\left(\mathrm{y}_{0}, \mathrm{y}_{1}, \mathrm{y}_{1}, \frac{\mathrm{t}}{\mathrm{q}^{\mathrm{n}}}\right)
\end{gathered}
$$

Then by the definition of IFMS,

$$
\begin{aligned}
\mathcal{M}\left(\mathrm{y}_{\mathrm{n}}, \mathrm{y}_{\mathrm{n}+\mathrm{p}}, \mathrm{y}_{\mathrm{n}+\mathrm{p}}, \mathrm{t}\right) & \geq \mathcal{M}\left(\mathrm{y}_{\mathrm{n}}, \mathrm{y}_{\mathrm{n}+1}, \mathrm{y}_{\mathrm{n}+1}, \frac{\mathrm{t}}{\mathrm{p}}\right) * \ldots \mathrm{p} \text { times } \ldots * \mathcal{M}\left(\mathrm{y}_{\mathrm{n}+\mathrm{p}-1}, \mathrm{y}_{\mathrm{n}+\mathrm{p}}, \mathrm{y}_{\mathrm{n}+\mathrm{p}}, \frac{\mathrm{t}}{\mathrm{p}}\right) \\
& \geq \mathcal{M}\left(\mathrm{y}_{0}, \mathrm{y}_{1}, \mathrm{y}_{1}, \frac{\mathrm{t}}{\mathrm{q}^{\mathrm{n}}}\right) * \ldots \mathrm{p} \text { times } \ldots * \mathcal{M}\left(\mathrm{y}_{0}, \mathrm{y}_{1}, \mathrm{y}_{1}, \frac{\mathrm{t}}{\mathrm{pq}^{\mathrm{n}+\mathrm{p}-1}}\right)
\end{aligned}
$$

Thus by the definition of IFMS,

$$
\begin{aligned}
\mathcal{N}\left(\mathrm{y}_{\mathrm{n}}, \mathrm{y}_{\mathrm{n}+\mathrm{p}}, \mathrm{y}_{\mathrm{n}+\mathrm{p}}, \mathrm{t}\right) & \leq \mathcal{N}\left(\mathrm{y}_{\mathrm{n}}, \mathrm{y}_{\mathrm{n}+1}, \mathrm{y}_{\mathrm{n}+1}, \frac{\mathrm{t}}{\mathrm{p}}\right) * \ldots \mathrm{p} \text { times } \ldots * \mathcal{N}\left(\mathrm{y}_{\mathrm{n}+\mathrm{p}-1}, \mathrm{y}_{\mathrm{n}+\mathrm{p}-1}, \mathrm{y}_{\mathrm{n}+\mathrm{p}}, \frac{\mathrm{t}}{\mathrm{p}}\right) \\
& \leq \mathcal{N}\left(\mathrm{y}_{0}, \mathrm{y}_{1}, \mathrm{y}_{1}, \frac{\mathrm{t}}{\mathrm{q}^{\mathrm{n}}}\right) * \ldots \mathrm{p} \text { times } \ldots * \mathcal{N}\left(\mathrm{y}_{0}, \mathrm{y}_{1}, \mathrm{y}_{1}, \frac{\mathrm{t}}{\mathrm{pq}^{\mathrm{n}+\mathrm{p}-1}}\right)
\end{aligned}
$$

$\lim _{\mathrm{n} \rightarrow \infty} \mathcal{M}\left(\mathrm{y}_{\mathrm{n}}, \mathrm{y}_{\mathrm{n}+\mathrm{p}}, \mathrm{y}_{\mathrm{n}+\mathrm{p}}, \mathrm{t}\right) \geq 1 * 1 * \ldots \mathrm{p}$ times $\ldots * 1 . \lim _{\mathrm{n} \rightarrow \infty} \mathcal{M}\left(\mathrm{y}_{\mathrm{n}}, \mathrm{y}_{\mathrm{n}+\mathrm{p}}, \mathrm{y}_{\mathrm{n}+\mathrm{p}}, \mathrm{t}\right)=1$ and

$\lim _{\mathrm{n} \rightarrow \infty} \mathcal{N}\left(\mathrm{y}_{\mathrm{n}}, \mathrm{y}_{\mathrm{n}+\mathrm{p}}, \mathrm{y}_{\mathrm{n}+\mathrm{p}}, \mathrm{t}\right) \leq 0 * 0 * \ldots * \mathrm{p}$ times $\ldots * 0 . \lim _{\mathrm{n} \rightarrow \infty} \mathcal{N}\left(\mathrm{y}_{\mathrm{n}}, \mathrm{y}_{\mathrm{n}+\mathrm{p}}, \mathrm{y}_{\mathrm{n}+\mathrm{p}}, \mathrm{t}\right)=0$

Thus $\left\{y_{n}\right\}$ is a Cauchy sequence in intuitionistic fuzzy metric space $X$.

Since $\mathrm{X}$ is complete, there exists a point $\mathrm{u} \in \mathrm{X}$ such that $\mathrm{y}_{\mathrm{n}} \rightarrow \mathrm{u}$.

Thus $\left\{\mathrm{ABx}_{2 \mathrm{n}}\right\},\left\{\mathrm{Qx}_{2 \mathrm{n}-1}\right\},\left\{\mathrm{STx}_{2 \mathrm{n}-1}\right\},\left\{\mathrm{Px}_{2 \mathrm{n}-2}\right\}$ are Cauchy sequence converge to $\mathrm{u}$.

Put $x=A B x_{2 n}, y=u, z=\operatorname{STx}_{2 n-1}$ in (iv), we get

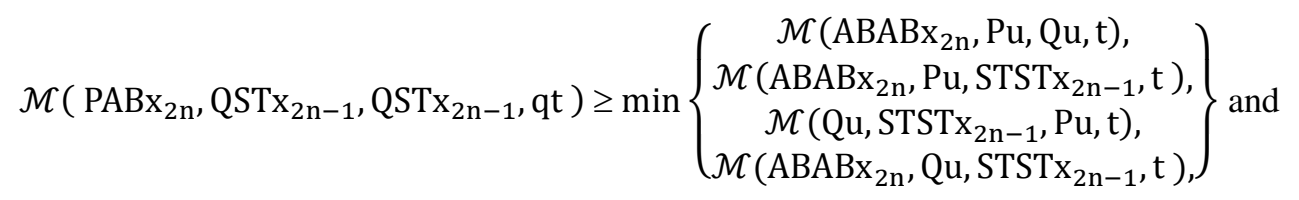

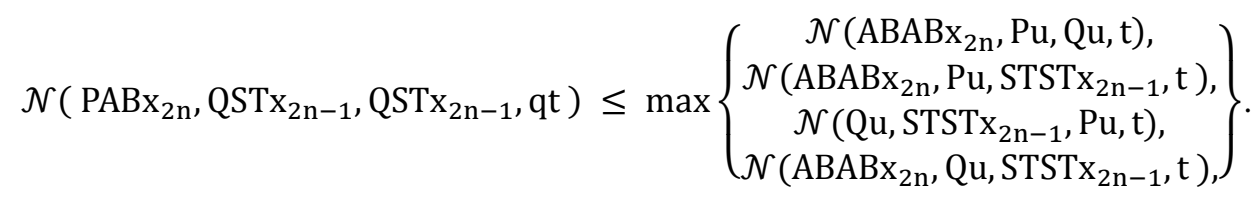


Now take the limit as $n \rightarrow \infty$ and using (ii), we get,

$$
\begin{gathered}
\mathcal{M}(\mathrm{Pu}, \mathrm{Qu}, \mathrm{Qu}, \mathrm{qt}) \geq \min \left\{\begin{array}{l}
\mathcal{M}(\mathrm{Pu}, \mathrm{Pu}, \mathrm{Qu}, \mathrm{t}), \mathcal{M}(\mathrm{Pu}, \mathrm{Pu}, \mathrm{Qu}, \mathrm{t}) \\
\mathcal{M}(\mathrm{Qu}, \mathrm{Qu}, \mathrm{Pu}, \mathrm{t}), \mathcal{M}(\mathrm{Pu}, \mathrm{Qu}, \mathrm{Qu}, \mathrm{t})
\end{array}\right\} \text { and } \\
\mathcal{N}(\mathrm{Pu}, \mathrm{Qu}, \mathrm{Qu}, \mathrm{qt}) \leq \max \left\{\begin{array}{l}
\mathcal{N}(\mathrm{Pu}, \mathrm{Pu}, \mathrm{Qu}, \mathrm{t}), \mathcal{N}(\mathrm{Pu}, \mathrm{Pu}, \mathrm{Qu}, \mathrm{t}) \\
\mathcal{N}(\mathrm{Qu}, \mathrm{Qu}, \mathrm{Pu}, \mathrm{t}), \mathcal{N}(\mathrm{Pu}, \mathrm{Qu}, \mathrm{Qu}, \mathrm{t})
\end{array}\right\} .
\end{gathered}
$$

Then by lemma 2.6, we get

$$
\begin{gathered}
\mathcal{M}(\mathrm{Pu}, \mathrm{Qu}, \mathrm{Qu}, \mathrm{qt}) \geq \mathcal{M}(\mathrm{Pu}, \mathrm{Qu}, \mathrm{Qu}, \mathrm{t}) \text { and } \\
\mathcal{N}(\mathrm{Pu}, \mathrm{Qu}, \mathrm{Qu}, \mathrm{qt}) \leq \mathcal{N}(\mathrm{Pu}, \mathrm{Qu}, \mathrm{Qu}, \mathrm{t})
\end{gathered}
$$

Therefore $\mathrm{Pu}=\mathrm{Qu}$. Now put $\mathrm{x}=\mathrm{ABx}_{2 \mathrm{n}}, \mathrm{y}=\mathrm{x}_{2 \mathrm{n}-1}, \mathrm{z}=\mathrm{x}_{2 \mathrm{n}-1}$, in (iv), we get

$$
\begin{gathered}
\mathcal{M}\left(\mathrm{PABx}_{2 \mathrm{n}}, \mathrm{Qx}_{2 \mathrm{n}-1}, \mathrm{Qx}_{2 \mathrm{n}-1}, \mathrm{qt}\right) \geq \min \left\{\begin{array}{c}
\mathcal{M}\left(\mathrm{ABABx}_{2 \mathrm{n}}, \mathrm{Px}_{2 \mathrm{n}-1}, \mathrm{Qx}_{2 \mathrm{n}-1}, \mathrm{t}\right) \\
\mathcal{M}\left(\mathrm{ABABx}_{2 \mathrm{n}}, \mathrm{Px}_{2 \mathrm{n}-1}, \mathrm{STx}_{2 \mathrm{n}-1}, \mathrm{t}\right), \\
\mathcal{M}\left(\mathrm{Qx}_{2 \mathrm{n}-1}, \mathrm{STx}_{2 \mathrm{n}-1}, \mathrm{Px}_{2 \mathrm{n}-1}, \mathrm{t}\right), \\
\mathcal{M}\left(\mathrm{ABABx}_{2 \mathrm{n}}, \mathrm{Qx}_{2 \mathrm{n}-1}, \mathrm{STx}_{2 \mathrm{n}-1}, \mathrm{t}\right)
\end{array}\right\} \text { and } \\
\mathcal{N}\left(\mathrm{PABx}_{2 \mathrm{n}}, \mathrm{Qx}_{2 \mathrm{n}-1}, \mathrm{Qx}_{2 \mathrm{n}-1}, \mathrm{qt}\right) \leq \max \left\{\begin{array}{c}
\mathcal{N}\left(\mathrm{ABABx}_{2 \mathrm{n}}, \mathrm{Px}_{2 \mathrm{n}-1}, \mathrm{Qx}_{2 \mathrm{n}-1}, \mathrm{t}\right) \\
\mathcal{N}\left(\mathrm{ABABx}_{2 \mathrm{n}}, \mathrm{Px}_{2 \mathrm{n}-1}, \mathrm{STx}_{2 \mathrm{n}-1}, \mathrm{t}\right), \\
\mathcal{N}\left(\mathrm{Qx}_{2 \mathrm{n}-1}, \mathrm{STx}_{2 \mathrm{n}-1}, \mathrm{Px}_{2 \mathrm{n}-1}, \mathrm{t}\right), \\
\mathcal{N}\left(\mathrm{ABABx}_{2 \mathrm{n}}, \mathrm{Qx}_{2 \mathrm{n}-1}, \mathrm{STx}_{2 \mathrm{n}-1}, \mathrm{t}\right)
\end{array}\right\}
\end{gathered}
$$

Thus we have $\mathcal{M}(\mathrm{Pu}, \mathrm{u}, \mathrm{u}, \mathrm{qt}) \geq \mathcal{M}(\mathrm{Pu}, \mathrm{u}, \mathrm{u}, \mathrm{t})$ and

$$
\mathcal{N}(\mathrm{Pu}, \mathrm{u}, \mathrm{u}, \mathrm{qt}) \geq \mathcal{N}(\mathrm{Pu}, \mathrm{u}, \mathrm{u}, \mathrm{t})
$$

Therefore $\mathrm{Pu}=\mathrm{u}$. This implies $\mathrm{Pu}=\mathrm{Qu}=\mathrm{u}$.

Now put $\mathrm{x}=\mathrm{Px}_{2 \mathrm{n}-2}, \mathrm{y}=\mathrm{Px}_{2 \mathrm{n}-2}, \mathrm{z}=\mathrm{u}$ in (iv), we get

$$
\mathcal{M}\left(\mathrm{PPx}_{2 \mathrm{n}-2}, \mathrm{Qu}, \mathrm{Qu}, \mathrm{qt}\right) \geq \min \left\{\begin{array}{c}
\mathcal{M}\left(\mathrm{ABPx}_{2 \mathrm{n}-2}, \mathrm{PPx}_{2 \mathrm{n}-2}, \mathrm{QPx} 2 \mathrm{P}-2, \mathrm{t}\right) \\
\mathcal{M}\left(\mathrm{ABPx}_{2 \mathrm{n}-2}, \mathrm{PPx}_{2 \mathrm{n}-2}, \mathrm{STu}, \mathrm{t}\right), \\
\mathcal{M}\left(\mathrm{QPx}_{2 \mathrm{n}-2}, \mathrm{STu}_{\mathrm{PPx}} \mathrm{PPn}_{2 \mathrm{n}}, \mathrm{t}\right), \\
\mathcal{M}\left(\mathrm{ABPx}_{2 \mathrm{n}-2}, \mathrm{QPx}_{2 \mathrm{n}-2}, \mathrm{STu}, \mathrm{t}\right)
\end{array}\right\} \text { and }
$$


Applied Mathematics and Sciences: An International Journal (MathSJ ), Vol. 2, No. 3, September 2015

$$
\mathcal{N}\left(\mathrm{PPx}_{2 \mathrm{n}-2}, \mathrm{Qu}, \mathrm{Qu}, \mathrm{qt}\right) \leq \max \left\{\begin{array}{c}
\mathcal{M}\left(\mathrm{ABPx}_{2 \mathrm{n}-2}, \mathrm{PPx}_{2 \mathrm{n}-2}, \mathrm{QPx} \mathrm{P}_{2 \mathrm{n}-2}, \mathrm{t}\right) \\
\mathcal{M}\left(\mathrm{ABPx}_{2 \mathrm{n}-2}, \mathrm{PPx}_{2 \mathrm{n}-2}, \mathrm{STu}, \mathrm{t}\right), \\
\mathcal{M}\left(\mathrm{QPx}_{2 \mathrm{n}-2}, \mathrm{STu}_{1} \mathrm{PPx}_{2 \mathrm{n}-2}, \mathrm{t}\right), \\
\mathcal{M}\left(\mathrm{ABPx}_{2 \mathrm{n}-2}, \mathrm{QPx}_{2 \mathrm{n}-2}, \mathrm{STu}, \mathrm{t}\right)
\end{array}\right\} .
$$

Now taking the limit as $n \rightarrow \infty$ and on using (ii) and (iii), we get

$$
\begin{aligned}
& \mathcal{M}(\mathrm{ABu}, \mathrm{u}, \mathrm{u}, \mathrm{qt}) \geq \min \left\{\begin{array}{c}
\mathcal{M}(\mathrm{ABu}, \mathrm{ABu}, \mathrm{u}, \mathrm{t}), \quad \mathcal{M}(\mathrm{ABu}, \mathrm{ABu}, \mathrm{u}, \mathrm{t}), \\
\mathcal{N}(\mathrm{Qu}, \mathrm{u}, \mathrm{ABu}, \mathrm{t}), \mathcal{M}(\mathrm{ABu}, \mathrm{Qu}, \mathrm{u}, \mathrm{t})
\end{array}\right\} \\
& \mathcal{N}(\mathrm{ABu}, \mathrm{u}, \mathrm{u}, \mathrm{qt}) \leq \max \left\{\begin{array}{c}
\mathcal{N}(\mathrm{ABu}, \mathrm{ABu}, \mathrm{u}, \mathrm{t}), \mathcal{N}(\mathrm{ABu}, \mathrm{ABu}, \mathrm{u}, \mathrm{t}), \\
\mathcal{N}(\mathrm{Qu}, \mathrm{u}, \mathrm{ABu}, \mathrm{t}), \mathcal{N}(\mathrm{ABu}, \mathrm{Qu}, \mathrm{u}, \mathrm{t})
\end{array}\right\} .
\end{aligned}
$$

This implies

$$
\begin{gathered}
\mathcal{M}(\mathrm{ABu}, \mathrm{u}, \mathrm{u}, \mathrm{qt}) \geq \min \left\{\begin{array}{c}
\mathcal{M}(\mathrm{ABu}, \mathrm{ABu}, \mathrm{u}, \mathrm{t}), \quad \mathcal{M}(\mathrm{ABu}, \mathrm{ABu}, \mathrm{u}, \mathrm{t}), \\
\mathcal{M}(\mathrm{u}, \mathrm{u}, \mathrm{ABu}, \mathrm{t}), \mathcal{M}(\mathrm{ABu}, \mathrm{u}, \mathrm{u}, \mathrm{t})
\end{array}\right\} \\
\mathcal{N}(\mathrm{ABu}, \mathrm{u}, \mathrm{u}, \mathrm{qt}) \leq \max \left\{\begin{array}{c}
\mathcal{N}(\mathrm{ABu}, \mathrm{ABu}, \mathrm{u}, \mathrm{t}), \mathcal{N}(\mathrm{ABu}, \mathrm{ABu}, \mathrm{u}, \mathrm{t}), \\
\mathcal{N}(\mathrm{u}, \mathrm{u}, \mathrm{ABu}, \mathrm{t}), \mathcal{N}(\mathrm{ABu}, \mathrm{u}, \mathrm{u}, \mathrm{t})
\end{array}\right\} .
\end{gathered}
$$

Therefore by lemma (2.6) we have $\mathrm{ABu}=\mathrm{u}$. Thus $\mathrm{Pu}=\mathrm{Qu}=\mathrm{ABu}=\mathrm{u}$.

Put $\mathrm{x}=\mathrm{u}, \mathrm{y}=\mathrm{u}, \mathrm{z}=\mathrm{Qx}_{2 \mathrm{n}-1}$, in (iv) we get

$$
\begin{aligned}
& \mathcal{M}\left(\mathrm{Pu}, \mathrm{QQx_{2n-1 }}, \mathrm{QQx_{2n-1 }}, \mathrm{qt}\right) \geq \min \left\{\begin{array}{c}
\mathcal{M}(\mathrm{u}, \mathrm{u}, \mathrm{u}, \mathrm{t}), \mathcal{M}(\mathrm{u}, \mathrm{u}, \mathrm{STu}, \mathrm{t}), \\
\mathcal{M}(\mathrm{u}, \mathrm{STu}, \mathrm{u}, \mathrm{t}), \mathcal{M}(\mathrm{u}, \mathrm{u}, \mathrm{STu}, \mathrm{t})
\end{array}\right\} \\
& \mathcal{N}\left(\mathrm{Pu}, \mathrm{QQx_{2n-1 }}, \mathrm{QQx}_{2 \mathrm{n}-1}, \mathrm{qt}\right) \leq \max \left\{\begin{array}{c}
\mathcal{N}(\mathrm{u}, \mathrm{u}, \mathrm{u}, \mathrm{t}), \mathcal{N}(\mathrm{u}, \mathrm{u}, \mathrm{STu}, \mathrm{t}), \\
\mathcal{N}(\mathrm{u}, \mathrm{STu}, \mathrm{u}, \mathrm{t}), \mathcal{N}(\mathrm{u}, \mathrm{u}, \mathrm{STu}, \mathrm{t})
\end{array}\right\},
\end{aligned}
$$

On using lemma, (2.6) we have

$$
\begin{gathered}
\mathcal{M}(\mathrm{STu}, \mathrm{STu}, \mathrm{u}, \mathrm{qt}) \geq \mathcal{M}(\mathrm{STu}, \mathrm{STu}, \mathrm{u}, \mathrm{t}) \text { and } \\
\mathcal{M}(\mathrm{STu}, \mathrm{STu}, \mathrm{u}, \mathrm{qt}) \geq \mathcal{M}(\mathrm{STu}, \mathrm{STu}, \mathrm{u}, \mathrm{t}) \\
\mathcal{N}(\mathrm{STu}, \mathrm{STu}, \mathrm{u}, \mathrm{qt}) \leq \mathcal{N}(\mathrm{STu}, \mathrm{STu}, \mathrm{u}, \mathrm{t})
\end{gathered}
$$

Thus $\mathrm{STu}=\mathrm{u}$. We get $\mathrm{Pu}=\mathrm{Qu}=\mathrm{ABu}=\mathrm{STu}=\mathrm{u}$.

\section{Uniqueness}

Let $w$ be another common fixed point of A, B, P, Q, S and T. Then $\mathcal{M}(\mathrm{Pu}, \mathrm{Qw}, \mathrm{Qw}, \mathrm{qt}) \geq \min \left\{\begin{array}{l}\mathcal{M}(\mathrm{ABu}, \mathrm{Pw}, \mathrm{Qw}, \mathrm{t}), \mathcal{M}(\mathrm{ABu}, \mathrm{Pw}, \mathrm{STw}, \mathrm{t}), \\ \mathcal{M}(\mathrm{Qw}, \mathrm{STw}, \mathrm{Pw}, \mathrm{t}), \mathcal{M}(\mathrm{ABu}, \mathrm{Qw}, \mathrm{STw}, \mathrm{t})\end{array}\right\}$ 
$\mathcal{M}(\mathrm{u}, \mathrm{w}, \mathrm{w}, \mathrm{qt}) \geq \min \left\{\begin{array}{l}\mathcal{M}(\mathrm{u}, \mathrm{w}, \mathrm{w}, \mathrm{t}), \mathcal{M}(\mathrm{u}, \mathrm{w}, \mathrm{w}, \mathrm{t}), \\ \mathcal{M}(\mathrm{w}, \mathrm{w}, \mathrm{w}, \mathrm{t}), \mathcal{M}(\mathrm{u}, \mathrm{w}, \mathrm{w}, \mathrm{t})\end{array}\right\}$

$\mathcal{M}(\mathrm{u}, \mathrm{w}, \mathrm{w}, \mathrm{qt}) \geq \mathcal{M}(\mathrm{u}, \mathrm{w}, \mathrm{w}, \mathrm{t})$ and

$\mathcal{N}(\mathrm{Pu}, \mathrm{Qw}, \mathrm{Qw}, \mathrm{qt}) \leq \max \left\{\begin{array}{l}\mathcal{N}(\mathrm{ABu}, \mathrm{Pw}, \mathrm{Qw}, \mathrm{t}), \mathcal{N}(\mathrm{ABu}, \mathrm{Pw}, \mathrm{STw}, \mathrm{t}), \\ \mathcal{N}(\mathrm{Qw}, \mathrm{STw}, \mathrm{Pw}, \mathrm{t}), \mathcal{N}(\mathrm{ABu}, \mathrm{Qw}, \mathrm{STw}, \mathrm{t})\end{array}\right\}$

$\mathcal{N}(\mathrm{u}, \mathrm{w}, \mathrm{w}, \mathrm{qt}) \leq \max \left\{\begin{array}{l}\mathcal{N}(\mathrm{u}, \mathrm{w}, \mathrm{w}, \mathrm{t}), \mathcal{N}(\mathrm{u}, \mathrm{w}, \mathrm{w}, \mathrm{t}), \\ \mathcal{N}(\mathrm{w}, \mathrm{w}, \mathrm{w}, \mathrm{t}), \mathcal{N}(\mathrm{u}, \mathrm{w}, \mathrm{w}, \mathrm{t})\end{array}\right\} \mathcal{N}(\mathrm{u}, \mathrm{w}, \mathrm{w}, \mathrm{qt}) \leq \mathcal{N}(\mathrm{u}, \mathrm{w}, \mathrm{w}, \mathrm{t})$,

which is a contradiction. Therefore $\mathrm{u}=\mathrm{w}$.

Hence the common fixed point is unique.

\section{Corollary 4.2}

Let $(\mathrm{X}, \mathcal{M}, \mathcal{N}, *, \diamond)$ be a complete generalized intuitionistic fuzzy metric space and let $\mathrm{A}, \mathrm{P}, \mathrm{Q}$ and $\mathrm{S}$ be self mappings of $\mathrm{X}$ satisfying the following conditions.

(i) $\quad \mathrm{P}(\mathrm{X}) \subseteq \mathrm{S}(\mathrm{X}), \mathrm{Q}(\mathrm{X}) \subseteq \mathrm{A}(\mathrm{X})$

(ii) The pair $(\mathrm{P}, \mathrm{A})$ and $(\mathrm{Q}, \mathrm{S})$ are compatible mappings of type $(\mathrm{P})$

(iii) $\mathrm{S}$ is continuous

(iv) $\mathcal{M}(\mathrm{Px}, \mathrm{Qz}, \mathrm{Qz}, \mathrm{qt}) \geq \min \{\mathcal{M}(\mathrm{Ax}, \mathrm{Py}, \mathrm{Qy}, \mathrm{t}), \mathcal{M}(\mathrm{Ax}, \mathrm{Py}, \mathrm{Sz}, \mathrm{t})$,

$\mathcal{M}(\mathrm{Qy}, \mathrm{Sz}, \mathrm{Py}, \mathrm{t}), \mathcal{M}(\mathrm{Ax}, \mathrm{Qy}, \mathrm{Sz}, \mathrm{t})\}$ and

$\mathcal{N}(\mathrm{Px}, \mathrm{Qz}, \mathrm{Qz}, \mathrm{qt}) \leq \max \{\mathcal{N}(\mathrm{Ax}, \mathrm{Py}, \mathrm{Qy}, \mathrm{t}), \mathcal{N}(\mathrm{Ax}, \mathrm{Py}, \mathrm{Sz}, \mathrm{t})$,

$\mathcal{N}(\mathrm{Qy}, \mathrm{Sz}, \mathrm{Py}, \mathrm{t}), \mathcal{N}(\mathrm{Ax}, \mathrm{Qy}, \mathrm{Sz}, \mathrm{t})\}$.

Then the mappings $\mathrm{P}, \mathrm{Q}, \mathrm{A}$ and $\mathrm{S}$ have a unique common fixed point in $\mathrm{X}$.

\section{Corollary 4.3}

Let $(\mathrm{X}, \mathcal{M}, \mathcal{N}, *, \diamond)$ be a complete generalized intuitionistic fuzzy metric space and let $\mathrm{B}, \mathrm{P}, \mathrm{Q}$ and $\mathrm{T}$ be self mappings of $\mathrm{X}$ satisfying the conditions (i), (ii), (iii), \& (iv) with $\mathrm{S}=\mathrm{I}$ and $\mathrm{A}=\mathrm{I}$;

Then the mappings $\mathrm{B}, \mathrm{P}, \mathrm{Q}$ and $\mathrm{T}$ have a unique common fixed point.

\section{Corollary 4.4}

Let ( $\mathrm{X}, \mathcal{M}, \mathcal{N}, *, \diamond$ ) be a complete generalized intuitionistic fuzzy metric space and let $\mathrm{A}, \mathrm{B}, \mathrm{P}, \mathrm{Q}, \mathrm{S}$ and $\mathrm{T}$ be self mappings of $\mathrm{X}$ satisfying the following conditions:

(i) $\quad \mathrm{P}(\mathrm{X}) \subseteq \mathrm{ST}(\mathrm{X}), \mathrm{Q}(\mathrm{X}) \subseteq \mathrm{AB}(\mathrm{X})$

(ii) The pair $(\mathrm{P}, \mathrm{AB})$ and $(\mathrm{Q}, \mathrm{ST})$ are compatible mappings of type $(\mathrm{P})$

(iii) $\mathrm{ST}$ is continuous

(iv) $\mathcal{M}(\mathrm{Px}, \mathrm{Qz}, \mathrm{Qz}, \mathrm{qt}) \geq \mathcal{M}(\mathrm{ABx}, \mathrm{Py}, \mathrm{Qy}, \mathrm{t}) * \mathcal{M}(\mathrm{ABx}, \mathrm{Py}, \mathrm{STz}, \mathrm{t}) *$

$\mathcal{M}(\mathrm{Qy}, \mathrm{STz}, \mathrm{Py}, \mathrm{t}) * \mathcal{M}(\mathrm{ABx}, \mathrm{Qy}, \mathrm{STz}, \mathrm{t})$ and

$\mathcal{N}(\mathrm{Px}, \mathrm{Qz}, \mathrm{Qz}, \mathrm{qt}) \leq \mathcal{N}(\mathrm{ABx}, \mathrm{Py}, \mathrm{Qy}, \mathrm{t}) \diamond \mathcal{N}(\mathrm{ABx}, \mathrm{Py}, \mathrm{STz}, \mathrm{t}) \diamond$

$\mathcal{N}(\mathrm{Qy}, \mathrm{STz}, \mathrm{Py}, \mathrm{t}) \diamond \mathcal{N}(\mathrm{ABx}, \mathrm{Qy}, \mathrm{STz}, \mathrm{t})$

Then the mappings $\mathrm{P}, \mathrm{Q}, \mathrm{AB}$ and $\mathrm{ST}$ have a unique common fixed point in $\mathrm{X}$. 


\section{REFERENCES}

1) Atanassov. K, Intuionistic fuzzy set and system, 29, (1986),87.

2) Banach. S, Theoric les operations linearires manograie, Mathmatyezne Warsaw, Poland, (1932)

3) Cho Y J, Pathak H K, Kang S M \& Jang J S, Common fixed points of compatible maps of type (b) of fuzzy metric spaces, fuzzy sets and systems 93, (1998) 99.

4) Choudhary B S, A unique common fixed point theorem for sequence of self maps in menger spaces, Bull. Korean math, Soc. 37 (2000). No. 3

5) Dahe, B.C ,Generalized metric spaces with fixed point, Bull.Calcutta Math.Soc.,84(4)(1992),107-113.

6) Deng Z K, Fuzzy pseudo- metric spaces, J. Math. Anal, Appl. 86 (1982) 74.

7) Fang J X, On fixed point theorems in fuzzy metric spaces, fuzzy sets and systems, 46,(1992) 107.

8) George A \& Veeramani P, On some results in fuzzy metric spaces, fuzzy sets and systems 64, (1994) $395-399$.

9) Grabiec M, Fixed points in fuzzy metric spaces, fuzzy sets and systems, 27(1988)385-389.

10) Gregori, VRomaguera. S. and Veereamani, P., A Note on Intuitionistic Fuzzy Metric Spaces, Chaos, Solitons and Fractals, vol.28, (2006) $902-905$.

11) Jungck, commuting mappings and fixed points, Amer. Math. Monthly, 83 (1976),261.

12) Kaleva O, Seikkala, S, On fuzzy metric spaces, Fuzzy sets and system, (1962) 122.

13) Kramosil I \& Michalek J, Fuzzy metric and statistical metric spaces, kyberntika Appl. 50, (1985)142.

14) Mishra S N, Sharma N \& Singh S I, common fixed point of maps on fuzzy metric spaces, Inter. J.Math. Sci. 17, (1994) 253.

15) Muthuraj. R, Pandiselvi .R, Common Fixed Point Theorems for compatible mappings of type (P-1) and type (P-2) in M- fuzzy metric spaces, Arab journal of mathematics \& mathematical science, Volume 3, No. 1-10(2013).

16) Park J H, Kwun Y C, \& Park J H, A fixed point theorem in the intuitionistic fuzzy metric spaces, far east J. Math. Sci. 16 (2005), 137-149.

17) Pathak H.K and M.S.Khan, A comparison of various types of Compatible maps and common fixed points, Indian J. Pure .appl.Math. 28(4).477-485 April 1977.

18) Surjith Singh Chauhan, Common Fixed Point Theorem for Two Pairs of Weakly Compatible Mappings in M -fuzzy Metric Spaces, Int.Journal of Math.Analysis, Vol.3, 2009, no.8,393-398.

19) S.Sedghi and N.Shobe, Fixed point theorem in M -fuzzy metric spaces with property (E), Advances in fuzzy Mathematics, 1(1) (2006), 55-65.

20) Surjeet singh chauhan \& Kiran utreja 2012, Common Fixed Point Theorem on M-fuzzy metric space using the concept of compatibility of type (P), Research Journal of Pure Algebra , 2(11), 2012, 350354

21) Turkoglu D, Altun I \& Cho Y J, Common fixed points of compatible mappings of type (I) and type (II) in fuzzy metric spaces, J. fuzzy math. 15(2007), 435.

22) Turkoglu D, Alaca C, Cho Y J \& Yildiz C, common fixed point theorems in intuitionistic fuzzy metric Spaces, J. Appl, Math, and computing 22(2006), 41.

23) Zadeh L A, Fuzzy sets, inform, and control 8 (1965) 338.]

\section{Authors}

Dr.R.Muthuraj received his Ph.D degree in Mathematics from Alagappa University Karaikudi, Tamil nadu, India in April 2010. Presently he is an Assistant Professor, PG \& Research Department of Mathematics, H.H.The Rajah's College, Pudukkottai Tamilnadu ,India. He has published over 80 papers in refereed National and International Journals. He is the reviewer and Editor of the reputed International Journals.Eight members are doing research work under his guidance. His research interests are Fuzzy Algebra, Lattice Theory, Discrete Mathematics, Fuzzy Topology, Fixed point theory and Fuzzy Graph Theory.

R. Pandiselvi received her M.Phil degree from School of Mathematics, Madurai Kamaraj University, Madurai, Tamilnadu, India. Now she is doing Ph.D at Bharathidasan University Tiruchirappalli,Tamilnadu, India. Presently she is working as an Associate Professor in Mathematics, The Madura college, Madurai, Tamilnadu, India. She has published over 10 papers in reputed National and International journals. Her research area is Fixed Point Theory.
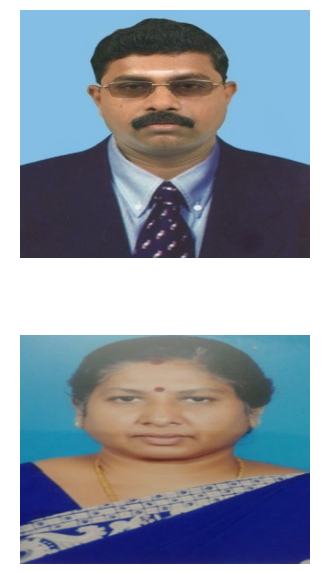